\title{
Retrospective validation of a new volumetric capnography parameter for the exclusion of pulmonary embolism at the emergency department
}

\author{
Timon M. Fabius (1) ${ }^{1,2}$, Michiel M.M. Eijsvogel (10 ${ }^{1}$, Marjolein G.J. Brusse-Keizer (10 ${ }^{3}$, \\ Olivier M. Sanchez ${ }^{4,5,6}$, Franck Verschuren ${ }^{7}$ and Frans H.C. de Jongh ${ }^{1,8}$
}

Affiliations: ${ }^{1}$ Dept of Pulmonology, Medisch Spectrum Twente, Enschede, The Netherlands. ${ }^{2}$ Dept of Research Methodology, Measurement and Data Analysis, Faculty of Behavioural, Management and Social Sciences, University of Twente, Enschede, The Netherlands. ${ }^{3}$ Medical School Twente, Medisch Spectrum Twente, Enschede, The Netherlands. ${ }^{4}$ Université Paris Descartes, Sorbonne Paris Cité, Paris, France. ${ }^{5}$ Service de Pneumologie et Soins intensifs, Hôpital Européen Georges Pompidou, Paris, France. ${ }^{6}$ INSERM UMR-S 1140 F-CRIN INNOVTE, Paris, France. ${ }^{7}$ Dept of Emergency Medicine, Cliniques Universitaire Saint-Luc, Université Catholique de Louvain, Brussels, Belgium. ${ }^{8}$ Engineering Technology Faculty, University of Twente, Enschede, The Netherlands.

Correspondence: Timon M. Fabius, Dept of Pulmonology, Medisch Spectrum Twente, Onderzoeksbureau Longgeneeskunde, P.O. Box 50000, 7500KA, Enschede, The Netherlands. E-mail: t.fabiusámst.nl

ABSTRACT Volumetric capnography might be used to exclude pulmonary embolism (PE) without the need for computed tomography pulmonary angiography. In a pilot study, a new parameter (CapNoPE) combining the amount of carbon dioxide exhaled per breath (carbon dioxide production $\left.\left(V \mathrm{CO}_{2}\right)\right)$, the slope of phase 3 of the volumetric capnogram (slope 3 ) and respiratory rate (RR) showed promising diagnostic accuracy (where CapNoPE $=\left(\mathrm{VCO}_{2} \times\right.$ slope 3$) / \mathrm{RR}$ ).

To retrospectively validate CapNoPE for the exclusion of PE, the volumetric capnograms of 205 subjects (68 with PE) were analysed, based on a large multicentre dataset of volumetric capnograms from subjects with suspected PE at the emergency department. The area under the curve (AUC) of the receiver operating characteristic (ROC) curve and diagnostic accuracy of the in-pilot established threshold (1.90 Pa.min) were calculated. CapNoPE was $1.56 \pm 0.97 \mathrm{~Pa} \cdot \mathrm{min}$ in subjects with PE versus $2.51 \pm 1.67 \mathrm{~Pa} \cdot \mathrm{min}$ in those without PE $(p<0.001)$. The AUC of the ROC curve was 0.714 (95\% CI 0.64-0.79). For the cut-off of $\geqslant 1.90 \mathrm{~Pa} \cdot \mathrm{min}$, sensitivity was $64.7 \%$, specificity was $59.9 \%$, the negative predictive value was $77.4 \%$ and the positive predictive value was $44.4 \%$.

The CapNoPE parameter is decreased in patients with PE but its diagnostic accuracy seems too low to use in clinical practice.

@ERSpublications

Retrospective validation in a large multicentre dataset shows that a novel volumetric capnography derived parameter (CapNoPE) is decreased in patients with PE but its diagnostic accuracy seems too low to use in clinical practice http://ow.ly/Ogpg $30 \mathrm{~m} 0 \mathrm{HuU}$

Cite this article as: Fabius TM, Eijsvogel MMM, Brusse-Keizer MGJ, et al. Retrospective validation of a new volumetric capnography parameter for the exclusion of pulmonary embolism at the emergency department. ERJ Open Res 2018; 4: 00099-2018 [https://doi.org/10.1183/23120541.000992018].

Received: June 282018 | Accepted after revision: Sept 182018

Copyright $\odot$ ERS 2018. This article is open access and distributed under the terms of the Creative Commons Attribution Non-Commercial Licence 4.0. 


\section{Introduction}

Pulmonary embolism (PE) has a high incidence and mortality is high when untreated [1]. The current gold standard to confirm or exclude the presence of emboli in the pulmonary arteries, computed tomography pulmonary angiography (CTPA), requires irradiation, is relatively expensive and cannot be directly applied in all patients seen in the emergency department (ED). Several strategies have been developed to reduce the number of CTPA scans needed. The most used strategy, to select the right patients for CTPA scanning, uses the Christopher algorithm and includes the use of a clinical prediction tool (such as the Wells score) and D-dimer testing [2]. Although its negative predictive value (NPV) is satisfying (enabling the safe exclusion of PE), its positive predictive value (PPV) is poor (i.e. less than $30 \%)[2,3]$. Therefore, the search for additional fast, cheap diagnostics to safely exclude PE in the ED without the need for CTPA continues.

The use of capnography in the exclusion of PE has been studied several times. Most of these studies focus on the usability of end-tidal carbon dioxide tension $\left(\mathrm{ETCO}_{2}\right)$, often combined with arterial carbon dioxide tension $\left(\mathrm{PaCO}_{2}\right)$, to calculate the alveolar dead-space fraction (AVDSf) which represents the fraction of ventilation that is wasted on dead space. Though the results of several individual studies seemed promising $[4,5]$, a meta-analysis showed a pooled sensitivity of $80 \%$ for both $\mathrm{PETCO}_{2}$ and AVDSf, and $73 \%$ for AVDSf alone [6]. Given the high mortality of untreated PE, these sensitivities are too low to justify the use of $\mathrm{PETCO}_{2}$ or AVDSf in clinical practice.

In volumetric capnography, the carbon dioxide tension $\left(\mathrm{PCO}_{2}\right)$ in exhaled air is plotted as a function of the exhaled volume (figure 1). The resulting curve is often divided into three phases: 1) the anatomic dead space (containing no carbon dioxide), 2) a transition phase (in which carbon dioxide increases rapidly), and 3) a plateau resembling alveolar air (in which a slight linear increase in carbon dioxide is seen due to continuous diffusion of carbon dioxide from the capillaries into the alveoli). Extensive reviews of volumetric capnography and its potential clinical applications have been produced $[7,8]$.

The volumetric capnogram and its phases contain more information than just $P_{\mathrm{ETCO}_{2}}$. It can be hypothesised that some (or a combination) of the volumetric capnography parameters are influenced by the presence of pulmonary emboli (and thus may be useful in the exclusion of PE). The number of studies investigating the usability of volumetric capnography in the exclusion of PE is limited. PATEL et al. [9] performed a neural network analysis on the volumetric capnogram of six subjects with PE and six subjects without PE. This analysis resulted in 17 volumetric capnography parameters which were associated with the presence of PE. In 1989, ERIKSsON et al. [10] proposed a novel parameter, the late dead-space fraction (Fdlate), where $F$ dlate=1-(extrapolated $\mathrm{PCO}_{2}$ at an exhaled volume of $15 \%$ of the predicted total lung capacity (TLC) $/ \mathrm{PaCO}_{2}$ ). They showed that Fdlate is increased in subjects with $\mathrm{PE}$ compared to healthy

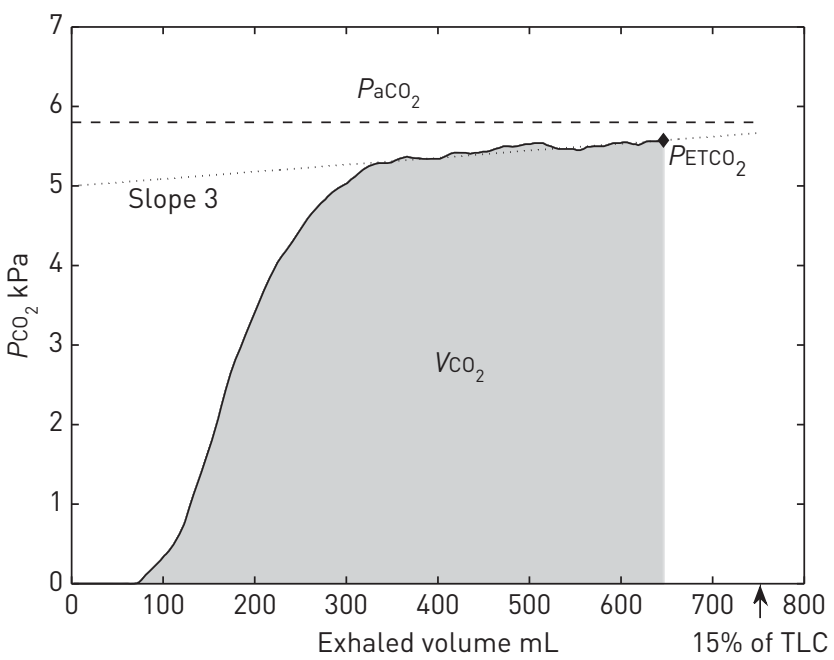

FIGURE 1 Example of a volumetric capnogram. A linear approximation of phase 3 of the capnogram (slope 3) is indicated by the dotted line. Carbon dioxide production $\left(\mathrm{V}_{\mathrm{CO}}\right)$ can be calculated by dividing the area under the curve $(A \cup C$ ) of the capnogram (indicated by the grey area) by the atmospheric pressure. Arterial carbon dioxide tension $\left(\mathrm{PaCO}_{2}\right)$ is indicated by the dashed line. Capnography variables were defined as follows: volumetric capnography parameter $(\mathrm{CapNoPE})=\left(\mathrm{VCO}_{2} \times\right.$ slope 3$) / \mathrm{RR}$; pulmonary embolism index (PE-index $)=$ $\left(\mathrm{PaCO}_{2}-\mathrm{PETCO}_{2}\right) /$ slope 3; late dead-space fraction $($ Fdlate $)=1$-(extrapolated $P_{\mathrm{CO}_{2}}$ at $15 \%$ of $\left.\mathrm{TLC} / \mathrm{PaCO}_{2}\right)$; alveolar dead-space fraction (AVDSf) $=\left(\mathrm{PaCO}_{2}-P_{\mathrm{ETCO}}\right) / \mathrm{PaCO}_{2}$. RR: respiratory rate; $P \mathrm{ETCO}_{2}$ : end-tidal carbon dioxide tension; $\mathrm{PCO}_{2}$ : carbon dioxide tension; TLC: total lung capacity 
subjects and subjects with obstructive pulmonary diseases. In 2004, VERSCHUREN et al. [11] compared the diagnostic accuracy of $F$ dlate, the arterial to end-tidal carbon dioxide gradient $\left(\mathrm{PaCO}_{2}-P \mathrm{ETCO}_{2}\right)$ and the slope of phase 3 (slope 3 ) of the volumetric capnogram (i.e. the alveolar phase). In this analysis, the Fdlate ratio seemed to have the highest diagnostic properties. The use of the slope of phase 3 seems physiologically justified as a decrease in pulmonary perfusion decreases slope 3. Some years later, VersChURen et al. [12] performed a larger study comparing Fdlate, AVDSf and a new parameter, pulmonary embolism index (PE-index), involving the arterial to end-tidal carbon dioxide gradient and the slope of phase 3, where PE-index $=\left(P_{\mathrm{aCO}_{2}}-\mathrm{PETCO}_{2}\right) /$ slope 3. Unfortunately, the volumetric capnography based parameters (PE-index and $F$ dlate) did not show better diagnostic properties when compared with the sole ratio of $\mathrm{PETCO}_{2}$ and $\mathrm{PaCO}_{2}$. In a recent pilot study [13] we proposed a novel volumetric capnography derived parameter, CapNoPE, which involves carbon dioxide production $\left(\mathrm{VCO}_{2}\right.$; the amount of carbon dioxide exhaled per breath), slope 3 and respiratory rate $(\mathrm{RR})$, such that $\mathrm{CapNoPE}=\left(V_{\mathrm{CO}} \times\right.$ slope 3$) / \mathrm{RR}$. As $\mathrm{PE}$ is likely to decrease slope 3 and $\mathrm{VCO}_{2}$, and might increase RR, we hypothesised that CapNoPE would be decreased in subjects with PE and would be independent of body weight (which is known to influence several capnography parameters). The pilot study results showed that CapNoPE was indeed significantly decreased in PE and a threshold of $1.90 \mathrm{~Pa} \cdot \mathrm{min}$ resulted in a sensitivity of $100 \%$ with a specificity of $47 \%$. However, given the limited number of subjects included, the 95\% CI values of the diagnostic accuracies were wide. As diagnostic properties are often overestimated in derivation sets, external validation is warranted [14]. The aim of the current study is to provide such validation by retrospectively assessing the diagnostic properties of CapNoPE for the exclusion of $\mathrm{PE}$ in data from the study performed by VERSCHUREN et al. [12].

\section{Methods}

The original study population consisted of subjects aged $>18$ years with suspected PE and elevated D-dimer levels at the ED of three academic hospitals in Brussels, Paris and Geneva, respectively and was part of a larger trial on the diagnosis of PE [15]. In this study, patients with suspected PE were randomised either to venous compression ultrasonography and, if negative, to CTPA, or to CTPA alone. PE was considered confirmed when shown on ultrasonography or CTPA, or when PE or deep vein thrombosis (DVT) were diagnosed within a follow-up period of 3 months.

The volumetric capnography measurements were performed between February 2005 and September 2006. Valid capnograms were obtained for 205 subjects and PE was diagnosed in 68 of them (33\%). Of these, only three were diagnosed by a positive venous compression ultrasonography finding alone. Among those in whom PE was excluded during initial presentation, none were diagnosed with any thromboembolic event during the 3 month follow-up period. At the time of measurement, the observer was unaware of the diagnosis. The original study was approved by the relevant ethical committees and all participating subjects provided written, informed consent.

The volumetric capnography data of the 205 subjects included were used to calculate CapNoPE. The diagnostic properties of CapNoPE were assessed using the area under the curve (AUC) of the receiver operating characteristic (ROC) curve and compared with the ROC curves of Fdlate, PE-index, AVDSf and $\mathrm{PETCO}_{2}$ alone. Furthermore, sensitivity, specificity, NPV and PPV were calculated for a CapNoPE threshold of $\geqslant 1.90 \mathrm{~Pa} \cdot \mathrm{min}$ to exclude PE.

Continuous variables were expressed as mean $\pm \mathrm{SD}$ or median (interquartile range (IQR)) as appropriate. Categorical variables were expressed as counts with corresponding percentages. Differences in continuous variables were tested using independent sample T-tests when normally distributed or Mann-Whitney U-tests when non-normally distributed. Differences in categorical variables were tested using the Chi-squared test or Fisher exact test. Test outcomes were considered significant for $\mathrm{p}<0.05$. All analyses were performed using IBM SPSS Statistics version 22 (IBM Corp, Armonk, NY, USA).

\section{Results}

Baseline characteristics of the 205 included subjects are provided in table 1. In total, 68 patients (33\%) had confirmed PE. Compared to those without PE, the subjects with PE had significantly higher clinical probabilities (as depicted by the revised Geneva scores) and higher D-dimer values. They were also longer in the ED before volumetric capnography measurements were performed. Detailed characteristics (e.g. comorbidities and presenting symptoms) are provided in the original publication by VeRSCHUREN et al. [12]. The outcomes of the several volumetric capnography variables are provided in table 2. All investigated capnography variables (CapNoPE, Fdlate, PE-index, AVDSf and $\mathrm{PETCO}_{2}$ ) were significantly different in the subjects with confirmed PE compared to those without PE. The ROC curves of these parameters are provided in figure 2. The AUC of the ROC curve for CapNoPE was 0.71 (95\% CI 0.64-0.79) and did not significantly differ from those of other parameters: PE-index 0.74 (95\% CI 0.67-0.82), AVDSf 0.73 


\begin{tabular}{|c|c|c|c|c|}
\hline \multirow[t]{2}{*}{ Variable } & \multirow[t]{2}{*}{ Subjects } & \multicolumn{2}{|c|}{ Diagnosis } & \multirow[t]{2}{*}{ p-value ${ }^{\#}$} \\
\hline & & $P E(n=68)$ & No PE (n=137) & \\
\hline Female & 108 (52.7) & 35 (51.5) & 73 (53.3) & 0.65 \\
\hline Age years & $65.1 \pm 16.8$ & $66.4 \pm 16.5$ & $64.4 \pm 16.9$ & 0.42 \\
\hline Height cm & $169 \pm 9$ & $170 \pm 10$ & $168 \pm 8$ & 0.21 \\
\hline Weight kg & $75.4 \pm 16.8$ & $76.0 \pm 14.4$ & $75.2 \pm 17.9$ & 0.76 \\
\hline Active smoker & $45(22.0)$ & $11(16.2)$ & $34(24.8)$ & 0.13 \\
\hline COPD & $24(11.7)$ & $5(7.4)$ & 19 (13.9) & 0.17 \\
\hline Previous PE & 38 (18.5) & $18(26.5)$ & $20(14.6)$ & 0.04 \\
\hline Heart failure & 17 (8.3) & 4 (5.9) & 13 (9.5) & 0.41 \\
\hline Cancer & $16(7.8)$ & $5(7.4)$ & $11(8.0)$ & 0.87 \\
\hline Heart rate beats $\cdot \min ^{-1}$ & $89 \pm 21$ & $94 \pm 22$ & $86 \pm 21$ & 0.01 \\
\hline Pulse oximetry $\%$ & 95 (92-97) & $94(90-96)$ & 96 (93-98) & 0.003 \\
\hline Thoracic pain & $124(60.5)$ & $39(57.4)$ & $85(62.0)$ & 0.48 \\
\hline Dyspnoea & $154(75.1)$ & $56[82.4]$ & 98 (71.5) & 0.11 \\
\hline Signs of DVT & 24 (11.7) & 10 (14.7) & $14(10.2)$ & 0.36 \\
\hline D-dimer level $\mu \mathrm{g} \cdot \mathrm{L}^{-1}$ & $1412(921-2926)$ & 3070 (1600-5117) & $1080(764-1929)$ & $<0.001$ \\
\hline Time in the ED $h$ & $5.0(3.0-11.0)$ & 5.5 (3.3-14.0) & $5.0(2.0-8.0)$ & 0.03 \\
\hline Symptoms h & 72 (24-168) & $72(24-168)$ & $72(24-180)$ & 0.91 \\
\hline Revised Geneva score & $5(3-7)$ & $6(5-8)$ & $4(3-6)$ & $<0.001$ \\
\hline \multicolumn{5}{|c|}{$\begin{array}{l}\text { Data are presented as } n(\%) \text {, mean } \pm \text { SD or median (interquartile range), unless otherwise stated. PE: } \\
\text { pulmonary embolism; COPD: chronic obstructive pulmonary disease; DVT: deep vein thrombosis; ED: } \\
\text { emergency department. \#: } p \text {-values are for differences between subjects with and without PE. Values in } \\
\text { bold are considered statistically significant }(p<0.05) \text {. }\end{array}$} \\
\hline
\end{tabular}

(95\% CI 0.65-0.80), Fdlate 0.69 (95\% CI 0.60-0.77) and $\mathrm{PETCO}_{2} 0.72$ (95\% CI 0.65-0.80) (figure 2). Cross-tabulation of the proposed CapNoPE threshold (1.90 Pa.min) against the diagnosis of $\mathrm{PE}$ is provided in table 3. A total of 106 subjects had a value of CapNoPE $\geqslant 1.90 \mathrm{~Pa} \cdot \mathrm{min}$, of which 24 were false-negatives. Of the 99 subjects with a value of CapNoPE $<1.90 \mathrm{~Pa} \cdot \mathrm{min}, 55$ were false-positives. This resulted in a sensitivity of $64.7 \%$ (95\% CI $52.2-75.9 \%$ ) with a specificity of $59.9 \%$ (95\% CI $51.1-68.1 \%$ ), a NPV of $77.4 \%$ (95\% CI $68.2-84.9 \%)$ and a PPV of $44.4 \%$ (95\% CI $34.5-54.8 \%)$.

TABLE 2 Values of the arterial blood gas and volumetric capnography variables in the groups with and without PE $(n=205)$

\begin{tabular}{|c|c|c|c|}
\hline \multirow[t]{2}{*}{ Variable } & \multicolumn{2}{|c|}{ Diagnosis } & \multirow[t]{2}{*}{ p-value ${ }^{\#}$} \\
\hline & $P E(n=68)$ & No PE (n=137) & \\
\hline $\mathrm{PaO}_{2} \mathrm{kPa}$ & $9.6 \pm 2.4$ & $10.4 \pm 2.5$ & 0.03 \\
\hline $\mathrm{PaCO}_{2} \mathrm{kPa}$ & $4.4 \pm 0.7$ & $4.6 \pm 0.7$ & 0.01 \\
\hline V $\mathrm{mL}$ & $694 \pm 303$ & $636 \pm 305$ & 0.20 \\
\hline RR breaths $\cdot \min ^{-1}$ & $18.9 \pm 5.9$ & $17.9 \pm 5.9$ & 0.23 \\
\hline $\mathrm{PETCO}_{2} \mathrm{kPa}$ & $3.41 \pm 0.89$ & $4.09 \pm 0.71$ & $<0.001$ \\
\hline Vaw/V \% & $38.9 \pm 8.7$ & $39.0 \pm 8.9$ & 0.96 \\
\hline $\mathrm{CCO}_{2} \mathrm{~mL} \cdot$ breath $^{-1}$ & $13.4 \pm 7.0$ & $14.9 \pm 9.1$ & 0.24 \\
\hline Slope $3 \mathrm{kPa} \cdot \mathrm{L}^{-1}$ & $1.94(1.06-3.39)$ & $2.70(1.54-5.24)$ & 0.001 \\
\hline PE-index $\mathrm{mL}$ & 422 (144-1048) & $146(67-286)$ & $<0.001$ \\
\hline Folate $\%$ & $0.73 \pm 45.7$ & $-15.2 \pm 34.1$ & $<0.001$ \\
\hline AVDSf $\%$ & $22.1 \pm 14.1$ & $11.3 \pm 9.9$ & 0.01 \\
\hline CapNoPE Pa.min & $1.56 \pm 0.97$ & $2.51 \pm 1.67$ & $<0.001$ \\
\hline \multicolumn{4}{|c|}{ 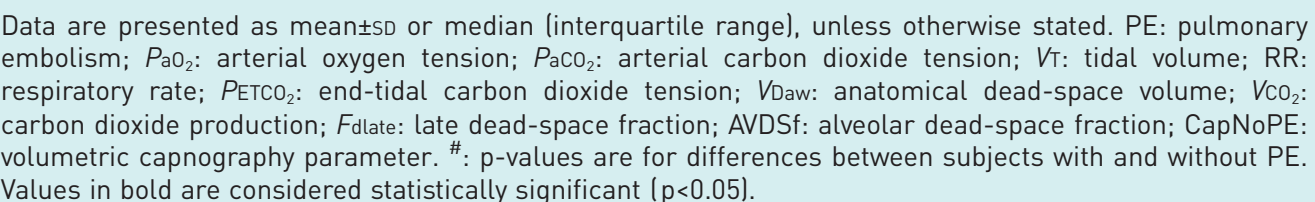 } \\
\hline
\end{tabular}


FIGURE 2 Receiver operating characteristic curves for five capnography parameters. CapNoPE: volumetric capnography parameter; AUC: area under the curve; $P \mathrm{ETCO}_{2}$ : end-tidal carbon dioxide tension; PE-index: pulmonary embolism index; Fdlate: late dead-space fraction; AVDSf: alveolar dead-space fraction.

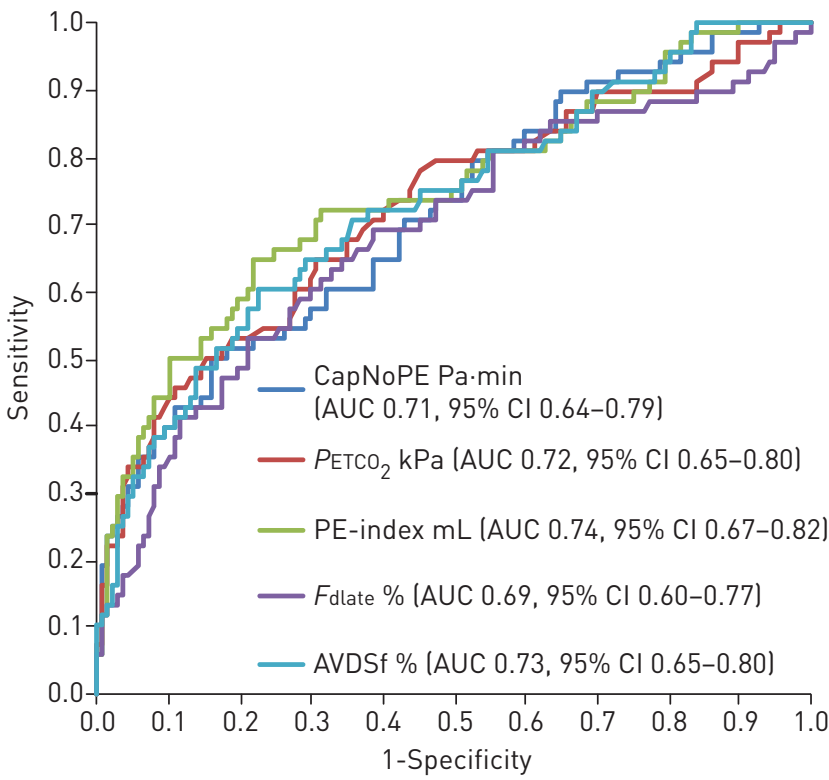

\section{Discussion}

The results confirm those of our pilot study which showed that CapNoPE is significantly decreased in patients with PE compared to those without. Furthermore, its diagnostic accuracy as expressed in the AUC of the ROC curve is similar to the earlier proposed parameters AVDSf, PE-index, Fdlate and $P_{\mathrm{ETCO}_{2}}$.

The large advantage of CapNoPE over AVDSf, PE-index and Fdlate is that it does not need arterial sampling and therefore is less burdensome for the patient. On the other hand, some form of dead-space fraction is simpler for clinicians to interpret, whereas CapNoPE represents an abstract entity. Furthermore, in the presence of respiratory comorbidities such as chronic obstructive pulmonary disease (COPD), arterial parameters might be essential for differentiation between the effects of PE and the effects of the comorbidity. Diseases such as COPD are known to alter the volumetric capnogram (mainly slope 3) [16] and might thus affect CapNoPE. Unfortunately, the number of subjects with COPD included was too low to investigate this potential influence. Moreover, $P \mathrm{ETCO}_{2}$ alone also does not need arterial sampling and its diagnostic accuracy is, in the current study population with a relatively low number of subjects with respiratory comorbidities, also comparable to the more advanced parameters.

The rationale behind this study is to find an easily accessible tool (in addition to the current diagnostic strategy) to exclude PE in the ED. Volumetric capnography is easy to apply in the ED and the cut-off for CapNoPE of $\geqslant 1.90$ Pa.min to exclude PE has shown promising sensitivity and specificity in a pilot study. However, the CIs of the diagnostic properties were wide, warranting further validation. Unfortunately, in the current larger dataset the proposed threshold results in a sensitivity $64.7 \%$ and a NPV of $77.4 \%$. There are no obvious differences in patient characteristics between the population data presented here and the pilot study that seem to explain the lower diagnostic properties. Of note, there were no differences in the distribution of CapNoPE between the data here and those of the pilot study ( $\mathrm{p}=0.22$ for subjects with PE and $\mathrm{p}=0.43$ for subjects without $\mathrm{PE}$ ). The development of CapNoPE was based on the hypothesis that $V \mathrm{CO}_{2}$ and slope 3 are decreased in the presence of PE whereas the RR is likely to increase. It was reasoned that the effects of $\mathrm{PE}$ on each parameter alone might be too small to detect and therefore they were combined to increase the differences. Indeed, the pilot study did not show significant differences in slope 3 ,

TABLE 3 Cross-tabulation of the proposed threshold (1.90 Pa.min) of the new volumetric capnography parameter (CapNoPE) against the diagnosis of pulmonary embolism (PE) ( $n=205$ )

\begin{tabular}{lccc} 
Parameter & \multicolumn{2}{c}{ Diagnosis } & Total \\
\cline { 2 - 4 } & PE & No PE & \\
CapNoPE $<1.90 \mathrm{~Pa} \cdot \mathrm{min}$ & 44 & 55 & 99 \\
CapNoPE $\geqslant 1.90 \mathrm{~Pa} \cdot \mathrm{min}$ & 24 & 82 & 106 \\
Total & 68 & 137 & 205
\end{tabular}


$V \mathrm{CO}_{2}$ or $\mathrm{RR}$ (although there was a non-significant trend towards higher $\mathrm{RR}$ and lower $V \mathrm{CO}_{2}$ in the subjects with PE), whereas the combination of these three parameters in CapNoPE was significantly decreased in PE subjects. The results presented here show no differences in either $\mathrm{RR}$ or $\mathrm{VCO}_{2}$ between the subjects with and without PE, but slope 3 is significantly decreased. The lower diagnostic properties found in the data presented here might be explained by the larger number of subjects included. As indicated earlier, the CIs of the diagnostic properties found in the pilot study were wide (due to a limited number of subjects).

Though the diagnostic properties of the proposed threshold are lower, it should be noted that the AUC of the ROC curve is well within the CI of the AUC found in the pilot study. However, given the potentially lethal consequences of PE, the NPV (77.4\%) is unacceptable for the safe exclusion of PE. The ROC curve also shows that the cut-offs with an acceptable NPV (>97\%) have poor specificity (and PPV $<30 \%)$. As the PPV of the current diagnostic strategy is already $20-30 \%$, these cut-offs would have no added value in the diagnostic workup of patients with suspected PE. This is also the case for the other capnography-derived parameters $P \mathrm{ETCO}_{2}, \mathrm{AVDSf}, \mathrm{PE}$-index and $F$ dlate. This seems to imply that volumetric capnography alone will not be sufficient to exclude PE. One reason for this might be the extent of some emboli. As the specifications of the imaging standards improve, smaller emboli are detected. Furthermore, the imaging standards detect the presence of clots in the pulmonary circulation, not their consequences on gas exchange. Another reason for the limited diagnostic capacity of capnography might be the high variability of breathing (and thus carbon dioxide exchange). However, prolonged periods of data were recorded and averaged, and the data was only used for analysis if variability was low (as pre-specified by several strict criteria, based on tidal volume $(V \mathrm{~T}), \mathrm{RR}$ and $\left.\mathrm{PETCO}_{2}\right)$ and visible inspection of the capnograms did not reveal obvious measurement errors [12]. This strategy is likely to have maximised the chance on representable measurements.

The current study has some limitations. As already mentioned CapNoPE is more abstract and therefore harder to interpret compared to dead-space fractions. However, all these parameters also require some level of physiologic expertise. The use of other tools to increase the PPV of the diagnostic workup of PE suspected patients, such as the age-adjusted D-dimer [17] or the Years algorithm [18], may therefore be more likely to succeed. The major limitation of this study is the relatively low number of subjects included. To be able to draw a definite conclusion on the usability of CapNoPE for the exclusion of PE, a much larger prospective trial would be required that included several respiratory and cardiovascular comorbidities. The definition of PE used in the original trial (positive CTPA or ultrasound at initial presentation, or a thromboembolic event during the 3 month follow-up period) might also appear to be a limitation. A positive venous ultrasonography finding alone, or the occurrence of a thromboembolic event during follow-up, does not necessarily imply that PE was present during the initial presentation when the volumetric capnography was performed. However, no thromboembolic events were recorded during the follow-up of subjects in whom PE was excluded at the initial presentation. Furthermore, PE was diagnosed based solely on a positive venous ultrasonography finding in only three subjects. Therefore it seems unlikely that this influenced the results of the current analysis.

\section{Conclusion}

The capnography-derived parameter CapNoPE is significantly decreased in subjects with PE compared to subjects without. Furthermore, its overall diagnostic properties are essentially equal to the diagnostic properties of $P_{\mathrm{ETCO}}$, PE-index, Fdlate and AVDSf. However, the NPV of the threshold value to exclude PE established earlier (CapNoPE $\geqslant 1.9 \mathrm{~Pa} \cdot \mathrm{min}$ ) seems too low to use in clinical practice.

Conflict of interest: T.M. Fabius has nothing to disclose. M.M.M. Eijsvogel has nothing to disclose. M.G.J. Brusse-Keizer has nothing to disclose. O.M. Sanchez reports grants and personal fees (for clinical trials) from Bayer, grants and other fees (for clinical trials) from Daiichi, grants from Actelion, grants and personal fees from MSD, and personal fees from BMS and Sanofi Aventis, outside the submitted work. F. Verschuren reports grants from Portola Pharmaceutical, Boehringer and Daiïchi, outside the submitted work. F.H.C. de Jongh has nothing to disclose.

Support Statement: The original study was supported by a grant from GE Healthcare.

\section{References}

1 Cohen AT, Agnelli G, Anderson FA, et al. Venous thromboembolism (VTE) in Europe. The number of VTE events and associated morbidity and mortality. Thromb Haemost 2007; 98: 756-764.

2 Van Belle A, Büller H, Huisman M, et al. Effectiveness of managing suspected pulmonary embolism using an algorithm combining clinical probability, D-dimer testing, and computed tomography. JAMA 2006; 295: 172-179.

3 Walen S, Leijstra MA, Uil SM, et al. Diagnostic yield of CT thorax angiography in patients suspected of pulmonary embolism: independent predictors and protocol adherence. Insights Imaging 2014; 5: 231-236. 
Kline JA, Meek S, Boudrow D, et al. Use of the alveolar dead space fraction (Vd/Vt) and plasma D-dimers to exclude acute pulmonary embolism in ambulatory patients. Acad Emerg Med 1997; 4: 856-863.

5 Yoon YH, Lee SW, Jung DM, et al. The additional use of end-tidal alveolar dead space fraction following D-dimer test to improve diagnostic accuracy for pulmonary embolism in the emergency department. Emerg Med J 2010; 27: $663-667$.

6 Manara A, D'hoore W, Thys F. Capnography as a diagnostic tool for pulmonary embolism: a meta-analysis. Ann Emerg Med 2013; 62: 584-591.

7 Suarez-Sipmann F, Bohm SH, Tusman G. Volumetric capnography: the time has come. Curr Opin Crit Care 2014; 20: $333-339$

8 Verscheure S, Massion PB, Verschuren F, et al. Volumetric capnography: lessons from the past and current clinical applications. Crit Care 2016; 20: 184.

9 Patel MM, Rayburn DB, Browning JA, et al. Neural network analysis of the volumetric capnogram to detect pulmonary embolism. Chest 1999; 116: 1325-1332.

10 Eriksson L, Wollmer P, Olsson CG, et al. Diagnosis of pulmonary embolism based upon alveolar dead space analysis. Chest 1989; 96: 357-362.

11 Verschuren F, Liistro G, Coffeng R, et al. Volumetric capnography as a screening test for pulmonary embolism in the emergency department. Chest 2004; 125: 841-850.

12 Verschuren F, Sanchez O, Righini M, et al. Volumetric or time-based capnography for excluding pulmonary embolism in outpatients? J Thromb Haemost 2010; 8: 60-67.

13 Fabius TM, Eijsvogel MM, van der Lee I, et al. Volumetric capnography in the exclusion of pulmonary embolism at the emergency department: a pilot study. J Breath Res 2016; 10: 46016.

14 Bleeker SE, Moll HA, Steyerberg EW, et al. External validation is necessary in prediction research: a clinical example. J Clin Epidemiol 2003; 56: 826-832.

15 Righini M, Le Gal G, Aujesky D, et al. Diagnosis of pulmonary embolism by multidetector CT alone or combined with venous ultrasonography of the leg: a randomised non-inferiority trial. Lancet 2008; 371: 1343-1352.

16 Long B, Koyfman A, Vivirito MA. Capnography in the emergency department: a review of uses, waveforms, and limitations. J Emerg Med 2017; 53: 829-842.

17 Righini M, Van Es J, Den Exter PL, et al. Age-adjusted D-dimer cutoff levels to rule out pulmonary embolism: the ADJUST-PE study. JAMA 2014; 311: 1117-1124.

18 van der Hulle T, Cheung WY, Kooij S, et al. Simplified diagnostic management of suspected pulmonary embolism (the YEARS study): a prospective, multicentre, cohort study. Lancet 2017; 390: 289-297. 